\title{
Kaiso differentially regulates components of the Notch signaling pathway in intestinal cells
}

Shaiya C. Robinson ${ }^{1}$, Kristina Klobucarr ${ }^{1,2}$, Christina C. Pierre ${ }^{1,3}$, Amna Ansari ${ }^{1}$, Svetlana Zhenilo ${ }^{4}$, Egor Prokhortchouk ${ }^{4}$ and Juliet M. Daniel ${ }^{1 *}$

\begin{abstract}
Background: In mammalian intestines, Notch signaling plays a critical role in mediating cell fate decisions; it promotes the absorptive (or enterocyte) cell fate, while concomitantly inhibiting the secretory cell fate (i.e. goblet, Paneth and enteroendocrine cells). We recently reported that intestinal-specific Kaiso overexpressing mice $\left(K_{\text {Kaiso }}{ }^{T \text { ) }}\right.$ ) exhibited chronic intestinal inflammation and had increased numbers of all three secretory cell types, hinting that Kaiso might regulate Notch signaling in the gut. However, Kaiso's precise role in Notch signaling and whether the Kaiso ${ }^{T g}$ secretory cell fate phenotype was linked to Kaiso-induced inflammation had yet to be elucidated.

Methods: Intestines from 3-month old Non-transgenic and Kaiso ${ }^{\text {Tg }}$ mice were "Swiss" rolled and analysed for the expression of Notch1, DIl-1, Jagged-1, and secretory cell markers by immunohistochemistry and immunofluorescence. To evaluate inflammation, morphological analyses and myeloperoxidase assays were performed on intestines from 3-month old Kaiso ${ }^{\text {Tg }}$ and control mice. Notch1, DIl-1 and Jagged-1 expression were also assessed in stable Kaisodepleted colon cancer cells and isolated intestinal epithelial cells using real time PCR and western blotting. To assess Kaiso binding to the DLL1, JAG1 and NOTCH1 promoter regions, chromatin immunoprecipitation was performed on three colon cancer cell lines.
\end{abstract}

Results: Here we demonstrate that Kaiso promotes secretory cell hyperplasia independently of Kaiso-induced inflammation. Moreover, Kaiso regulates several components of the Notch signaling pathway in intestinal cells, namely, DII-1, Jagged-1 and Notch1. Notably, we found that in Kaiso ${ }^{\text {Tg }}$ mice intestines, Notch1 and DII-1 expression are significantly reduced while Jagged-1 expression is increased. Chromatin immunoprecipitation experiments revealed that Kaiso associates with the DLL1 and JAG1 promoter regions in a methylation-dependent manner in colon carcinoma cell lines, suggesting that these Notch ligands are putative Kaiso target genes.

Conclusion: Here, we provide evidence that Kaiso's effects on intestinal secretory cell fates precede the development of intestinal inflammation in Kaiso ${ }^{\text {Tg }}$ mice. We also demonstrate that Kaiso inhibits the expression of DII-1, which likely contributes to the secretory cell phenotype observed in our transgenic mice. In contrast, Kaiso promotes Jagged-1 expression, which may have implications in Notch-mediated colon cancer progression.

Keywords: Kaiso, Notch, DII-1, Jagged-1, Intestinal homeostasis, Intestinal cell fates

\footnotetext{
*Correspondence: danielj@mcmaster.ca

'Department of Biology, McMaster University, Hamilton L8S 4K1, ON, Canada

Full list of author information is available at the end of the article
} International License (http://creativecommons.org/licenses/by/4.0/), which permits unrestricted use, distribution, and reproduction in any medium, provided you give appropriate credit to the original author(s) and the source, provide a link to the Creative Commons license, and indicate if changes were made. The Creative Commons Public Domain Dedication waiver (http://creativecommons.org/publicdomain/zero/1.0/) applies to the data made available in this article, unless otherwise stated. 


\section{Background}

The mammalian intestine is a rapidly self-renewing epithelium that undergoes continual turnover every 35 days $[1,2]$. The mucosa of the small intestine is folded into finger-like projections known as villi [1], which serve to maximize the surface area over which nutrient uptake occurs [3]. Most intestinal epithelial cells (IECs) migrate toward the tip of the villus, where they undergo apoptosis and are shed into the intestinal lumen [4]. The majority of IECs in the intestinal tract consists primarily of absorptive cells known as enterocytes. The remaining cells are categorized as secretory cells and include mucus-secreting goblet cells, hormonesecreting enteroendocrine cells, and anti-microbial Paneth cells [1, 3-5]. Unlike most terminally differentiated IECs, Paneth cells migrate downward toward the base of intervillar crypts, where intestinal stem and progenitor cell populations also reside [3, 6, 7]. The constant turnover of cells within the intestinal epithelium requires strict regulation of the signaling pathways that govern stem and progenitor cell proliferation and differentiation. While several pathways are critical for intestinal homeostasis, the Notch signaling pathway is indispensible for dictating intestinal cell fate decisions [8-13].

Notch receptors are heterodimeric single-pass transmembrane proteins [10, 14-16] with four receptors present in mammals (Notch1-4), each encoded by a separate gene [15]. Active Notch signaling is initiated upon binding of the Notch receptor to the transmembrane Delta/Serrate/Lag2 (DSL) family of Notch ligands, including Delta-like ligand (Dll)-1, Dll-4 and Jagged-1 $[15,16]$. Activated Notch then undergoes multiple proteolytic cleavages, which culminates in the cytoplasmic release of the Notch intracellular domain (NICD) fragment [16]. NICD subsequently translocates to the nucleus and interacts with RBPJ (recombination signal binding protein for immunoglobulin kappa J region; also known as CSL - CBF1, suppressor of hairless, Lag2) to transactivate the expression of Notch target genes, most notably, the Hes (Hairy enhancer of split) family of transcriptional repressors $[9,10,14]$.

In the intestines, Notch signaling dictates binary cell fate decisions - progenitor cells that lack Notch signaling are fated toward the secretory cell lineage (i.e. goblet, Paneth and enteroendocrine cells), while those with active Notch signaling are fated to become enterocytes [17]. Thus, misregulated Notch signaling in the intestines perturb cell fate decisions. For example, loss of Dll1 and combined loss of Hes1, Hes3 and Hes5 result in increased goblet, Paneth and enteroendocrine cells $[8,13,18]$. We recently reported that ectopic expression of the poxvirus and zinc finger (POZ)-zinc finger (hereafter, POZ-ZF) transcription factor Kaiso in the intestines of 12-month old mice (Kaiso $^{T g}$ ) resulted in chronic intestinal inflammation and a significant increase in secretory cell types compared to nontransgenic (NonTg) mice. This finding, coupled with our observation that expression of the Notch target gene Hes1 was reduced in Kaiso ${ }^{T g}$ compared to NonTg mice, implicated Kaiso as a negative regulator of Notch signaling [19].

Since Kaiso overexpression in 12-month old mice is reminiscent of loss of Notch pathway activity, we sought to further investigate Kaiso's role in Notch-mediated intestinal homeostasis and cell fate decisions. We found that the Kaiso-induced increase in intestinal secretory cells occurs prior to the onset of chronic intestinal inflammation, suggesting that the secretory cell phenotype does not manifest as a consequence of Kaiso-induced chronic inflammation. Notably, we found that Kaiso inhibits Dll-1 expression in the intestine, and we postulate that this inhibition contributes to the Kaiso-induced increase in secretory cell types. Surprisingly however, we found that Kaiso promotes Jagged-1 expression, which has been previously implicated in colon cancer progression [20-23]. Collectively, these data highlight novel roles for Kaiso in regulating Notch-mediated intestinal homeostasis.

\section{Methods \\ Mouse husbandry of Kaiso ${ }^{T g}$ tissues}

All mice were fed a standard chow diet and maintained in a specific pathogen-free room on a 12-h light/dark cycle. Kaiso ${ }^{T g}$ mice were identified by genotyping using PCR analysis of DNA isolated from ear snips. All PCR primers used are listed in Table 1. All animals were sacrificed by $\mathrm{CO}_{2}$ asphyxiation and cervical dislocation.

\section{Kaiso null tissues}

Kaiso null $\left(\mathrm{Kaiso}^{-/ y}\right)$ intestinal tissues were kindly provided by Dr. Egor Prokhortchouk. Intestinal tissues were fixed in Carnoy's fixative $(60 \% \mathrm{EtOH}, 30 \%$ chloroform, $10 \%$ acetic acid) for $16 \mathrm{~h}$ at $4{ }^{\circ} \mathrm{C}$, dehydrated and then paraffin embedded for immunohistochemistry [24].

\section{Cell culture}

HCT116, HT29, and SW480 human colon cancer cells were purchased from the American Tissue Culture Collection. All cells were cultured in Dulbecco's Modified Eagle's Medium supplemented with $10 \%$ fetal bovine serum and 1\% antibiotic-antimycotic (Thermo Fisher Scientific). Stable HCT116 and HT29 cell lines containing the retroviral pRS-shKaiso or pRS-shKaisoscrambled sequence [25], were selected with $2 \mu \mathrm{g} / \mathrm{mL}$ puromycin, while stable SW480 cells were selected with $4 \mu \mathrm{g} / \mathrm{mL}$ puromycin. All cells were grown and maintained at $37{ }^{\circ} \mathrm{C}, 5 \% \mathrm{CO}_{2}$. 
Table 1 List of primer sequences used for genotyping, ChIP-PCR and GRT-PCR

\begin{tabular}{|c|c|c|}
\hline Experiment & Target & Primer sequence $5^{\prime}-3^{\prime}$ \\
\hline \multirow[t]{2}{*}{ Genotyping } & fSV40-Kaiso & ATCATCAAAGCCGGGTGGGCA \\
\hline & rSV40-Kaiso & TITTCTACTCTCCATTTCATTCAAGTCCTC \\
\hline \multirow[t]{6}{*}{ ChIP } & DLL1KBS F & AAGCTCTGCAGCTCTCTTGG \\
\hline & DLL1KBS R & GGCGACTTTCGTITTCCTC \\
\hline & JAG1KBS B F & AGCTCTTGTGGCCTCACTTC \\
\hline & JAG1KBS B R & CCTCAGGCACTACCTCCAGA \\
\hline & JAG1KBS C F & CCTGAGGGTGTAAGTGATAGGC \\
\hline & JAG1KBS C R & GAGGAAAGGGAAATGTTGGG \\
\hline \multirow[t]{16}{*}{ qRT-PCR } & $m D \|-1 F$ & GCGACTGAGGTGTAAGATGGAA \\
\hline & $m D \|-1 R$ & TCTCAGCAGCATTCATCGGG \\
\hline & $\mathrm{mDll}-4 \mathrm{~F}$ & GCAAACTGCAGAACCACACA \\
\hline & $m D \|-4 R$ & TGGCTTCTCACTGTGTAACCG \\
\hline & mNotch1 F & ACAGTGCAACCCCCTGTATG \\
\hline & mNotch1 R & TCTAGGCCATCCCACTCACA \\
\hline & mNotch2 F & ACAGTGTTGGCTCCCTGTTC \\
\hline & mNotch2 R & ATCGTTACCTTGCCAGCCA \\
\hline & mHMBS F & GATGGGCAACTGTACCTGACTG \\
\hline & mHMBS R & CTGGGCTCCTCTTGGAATG \\
\hline & hDll1 F & AGAAAGTGTGCAACCCTGGC \\
\hline & hDII-1 R & CACTCTGCACTTGCATTCCCC \\
\hline & hJag1 F & CGCAAGCGATGTAGATTGAATATT \\
\hline & hJag1 R & CGCAAGCGATGTAGATTGAATATT \\
\hline & $\beta$-Actin F & СTCTTCCAGCCTTCCTTCCT \\
\hline & $\beta$-Actin R & AGCACTGTGTTGGCGTACAG \\
\hline
\end{tabular}

\section{Immunohistochemistry}

Kaiso $^{T g}$ intestinal tissues were formalin-fixed and paraffin embedded as previously described [19]. Periodic acid-Schiff (PAS) staining was performed by the John Mayberry Histology Facility at McMaster University. Immunohistochemistry (IHC) analysis of all other protein targets was performed as previously described [19], with the following modifications: antigen retrieval for chromogranin A was accomplished by heating tissues in $10 \mathrm{mM}$ sodium citrate, $\mathrm{pH} 6.0$ for $10 \mathrm{~min}$ at sub-boiling temperature; retrieval for NICD, Dll-1, Dll-4, Hes1, and Hes5 was accomplished by heating tissues at sub-boiling temperature for $15 \mathrm{~min}$ in TE-Tween (Tris EDTA, 0.05\% Tween), $\mathrm{pH}$ 9.0; and retrieval for lysozyme was performed with $200 \mu \mathrm{g} / \mathrm{mL}$ proteinase $\mathrm{K}, 50 \mathrm{mM}$ Tris $\mathrm{pH} 7.4$ at RT for $5 \mathrm{~min}$. Tissues were incubated with the following primary antibodies overnight at $4{ }^{\circ} \mathrm{C}$ at the indicated dilutions: rabbit anti-lysozyme (Thermo Scientific cat. \#PA1-29680; 1:50); rabbit antichromogranin A (Abcam cat. \#ab15160; 1:500); rabbit anti-Cleaved Notch 1 Val-1744 (Cell Signaling Technology cat. \#4147; 1:75); rabbit anti-Hes1 (Cell
Signaling Technology cat. \#11988S; 1:80); rabbit antiHes5 (Abcam cat. \#ab65077; 1:125); rabbit anti-Dll-1 (Abcam cat. \#ab84620; 1:100); and goat anti-Dll-4 (R\&D Systems cat. \#AF1389).

\section{Immunofluorescence}

For fluorescent staining of Jagged-1, tissue sections were prepared as described for IHC, however antigen retrieval was accomplished by treatment with $20 \mu \mathrm{g} / \mathrm{mL}$ proteinase $\mathrm{K}$ diluted in TE, pH 9.0 for $20 \mathrm{~min}$ at $37^{\circ} \mathrm{C}$. Following incubation with primary antibody (rabbit anti-Jagged-1, Santa Cruz cat. \#sc-8303; 1:100) and subsequent washes, tissues were incubated with a 1:500 dilution of secondary anti-rabbit Alexa-546 (Life Technologies cat. \#A11010) for $2 \mathrm{~h}$ at room temperature (RT). Tissues were then washed 3 times for $10 \mathrm{~min}$ with $0.05 \%$ Tris buffered saline (TBS)Tween, once for $5 \mathrm{~min}$ with TBS, and counterstained with TOTO-3 iodide (Life Technologies cat. \#T3604; 1:2000) for $30 \mathrm{~min}$ at RT. Excess TOTO-3 was removed and slides were allowed to dry prior to mounting with Prolong ${ }^{\circledR}$ Gold anti-fade reagent (Life Technologies cat. \#P10144).

\section{Protein isolation Intestinal epithelial cell protein isolation}

Small intestines were harvested and flushed with icecold saline solution $(150 \mathrm{mM} \mathrm{NaCl}, 2 \mathrm{mM}$ imidazole, $0.02 \% \mathrm{NaN}_{3}$ ), cut into $1-2 \mathrm{~cm}$ pieces and stirred vigorously in ice-cold sucrose solution (12 mM EDTA,

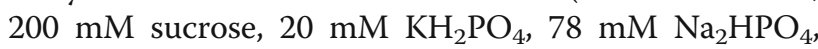
$0.02 \% \mathrm{NaN}_{3}$ ) for $2 \mathrm{~h}$ at $4{ }^{\circ} \mathrm{C}$. IECs were separated from large tissue segments using a metal strainer, and then pelleted at $200 \mathrm{x} \mathrm{g}$ for $10 \mathrm{~min}$ at $4{ }^{\circ} \mathrm{C}$. The cell pellet was washed 3 times with sucrose buffer, lysed in Laemmli sample buffer (LSB, 2\% SDS, 5\% glycerol, 62.5 $\mathrm{mM}$ Tris, $\mathrm{pH}$ 6.8) containing cOmplete Mini protease inhibitor cocktail (PIC) tablet (Roche cat. \#11836153001) on ice for $15 \mathrm{~min}$ with periodic vortexing, and denatured by boiling for $5 \mathrm{~min}$.

\section{Cultured cell protein isolation}

Cells were washed twice with cold $1 \mathrm{X}$ phosphate buffered saline (PBS) and pelleted at $1000 \mathrm{RPM}$ at $4{ }^{\circ} \mathrm{C}$ for 5 min. Cell pellets were lysed with LSB containing PIC on ice for 15 min with periodic vortexing and denatured by boiling for $5 \mathrm{~min}$. Denatured lysates were centrifuged at $13,200 \mathrm{RPM}, 4{ }^{\circ} \mathrm{C}$ for $15 \mathrm{~min}$; supernatants were transferred to new pre-chilled tubes and quantified by DC Protein Assay (Bio-Rad cat. \#500-0116).

\section{Myeloperoxidase (MPO) assay}

MPO activity of $50 \mathrm{mg}$ of flash frozen ileum was analyzed as previously described $[19,26]$. Briefly, tissues were homogenized in $0.5 \%$ HTAB (hexadecyltrimethylammonium bromide) buffer via sonication at $30 \mathrm{~Hz}$ 
for $4 \mathrm{~min}$, and centrifuged at 12,000 RPM for $15 \mathrm{~min}$ at $4{ }^{\circ} \mathrm{C}$. o-dianisidine dihydrochloride solution was added to the homogenates in triplicate in 96-well plates, and absorbance was measured at $450 \mathrm{~nm}$ every $30 \mathrm{~s}$ for $90 \mathrm{~s}$, in triplicate readings.

\section{SDS-PAGE and Immunoblotting}

Equal amounts of protein of interest were separated by SDS-PAGE and transferred onto a nitrocellulose membrane. Membranes were blocked with 3\% milk diluted in 1XTBS for $1 \mathrm{~h}$ at RT, with the exception of membranes probed with anti-cleaved Notch1 Val-1744, which were blocked with $5 \%$ BSA in $0.1 \%$ TBS-Tween20. Membranes were incubated overnight at $4{ }^{\circ} \mathrm{C}$ with primary antibodies at the following dilutions: rabbit anti-Kaiso (1:5000); rabbit anti-Cleaved Notch1 Val-1744 (Cell Signaling Technologies; 1:1000); rabbit anti-Dll-1 (Abcam; 1:500); mouse anti- $\beta$-actin (Sigma Aldrich cat\#. A5441-.2ML; 1:50,000). Membranes were then washed 5 times for 5 min with 1XTBS, incubated with the appropriate HRP-conjugated secondary antibody for $2 \mathrm{~h}$ at RT, and then washed 5 times for $5 \mathrm{~min}$ with 1XTBS. Proteins were visualized using Clarity Western ECL Substrate (Bio-Rad cat. \#170-5060) and images were acquired using the Bio-Rad ChemiDoc.

\section{Quantitative real-time reverse transcription PCR}

Total RNA was isolated using the NucleoSpin RNA kit (Macherey-Nagel cat. \#740984.250) according to manufacturer's protocols. mRNA was converted to cDNA using the SensiFAST cDNA Synthesis Kit, according to the manufacturer's instructions (Bioline cat. \#BIO65054) and qRT-PCR analysis was performed using the SensiFAST SYBR Hi-ROX Kit, (Bioline cat. \#BIO92020). Gene expression changes were normalized to $\beta$ actin (cultured cells) or hydroxymethylbilane synthase (HMBS; intestinal epithelial cells), and quantified using the standard curve method. Statistical calculations were performed using Student's t-test, and a $p$-value $\leq 0.05$ was considered statistically significant. The primer sequences used are listed in Table 1.

\section{Chromatin immunoprecipitation (ChIP)}

Chromatin was isolated as previously described [27]. For de-methylation studies, cells were treated with $5 \mu \mathrm{M} \mathrm{5}$ 'aza-cytidine (5'-aza; Sigma Aldrich; cat. \# A2385100MG) every $17 \mathrm{~h}$ for 5 consecutive days. Fifteen micrograms of chromatin was precipitated with $8 \mu \mathrm{g}$ mouse anti-Kaiso 6F monoclonal [28], $4 \mu \mathrm{g}$ mouse non-specific IgG (Abcam cat. \#ab37415) or $4 \mu \mathrm{g}$ rabbit anti-Histone H3 (Abcam cat. \#ab1791) antibodies. Precipitated DNA fragments were resuspended with nuclease-free water and PCR amplified. Primers used for ChIP are listed in Table 1.

\section{CpG Island prediction}

Potential transcription start sites (TSS) were obtained from the Eukaryotic Promoter Database [29]. DNA sequences at the indicated ranges surrounding the TSS were analyzed using the MethPrimer [30] and EMBOSS Cpgplot [31] programs to predict CpG islands.

\section{Promoter-reporter assays}

To create the pGLuc-4XCSL reporter construct, the 4XCSL sites were subcloned from the pGL2-4XCSLluciferase vector (a gift from Raphael Kopan; Addgene plasmid \#41726) [32], into pGLuc-basic (New England Biolabs) using EcoRI and HindIII. The pCAGGS-NICD expression vector was a gift from Nicholas Gaiano (Addgene plasmid \#26891) [33]. SW480 and HCT116 cells were seeded in technical triplicate into 6-well dishes at a density of $4 \times 10^{5}$ cells/well. Approximately 12-16 h post-seeding, cells were co-transfected with $1.0 \mu \mathrm{g}$ pGLuc-4XCSL, $0.5 \mu \mathrm{g}$ pRS- $\beta$-galactosidase, $0.175 \mu \mathrm{g}$ pCAGGS-NICD, and the indicated amount of pcDNA3-hKaiso using TurboFect ${ }^{\text {ti }}$ Transfection Reagent (Thermo Fisher cat. \#R0532). Twenty-four hours posttransfection, culture media was assayed for luciferase using the Biolux ${ }^{\text {mix }}$ Gaussia Luciferase Assay Kit (New England Biolabs; cat. \#E3300L), and read on an LB luminometer (Thermo Fisher). Luciferase activity was normalized to $\beta$-galactosidase to control for transfection efficiency. The average luciferase values from 3 biological replicates were calculated and plotted as relative fold change against the empty pGLuc-basic vector. Statistical significance was calculated using one-way analysis of variance (ANOVA).

\section{Results}

Ectopic Kaiso expression decreases Notch signaling and increases secretory cell numbers independent of Kaisoinduced inflammation.

Our finding that Kaiso overexpression in the murine gut (Kaiso $^{T g}$ ) results in chronic intestinal inflammation and an increase in secretory cells in 12-month (mo.) old mice compared to age-matched NonTg mice [19], was paradoxical since chronic inflammation is typically associated with a reduction in goblet cells $[34,35]$. Thus, we sought to determine whether the Kaiso ${ }^{T g}$ secretory cell phenotype occurred independently, or as a consequence of the Kaiso-induced chronic inflammation by analyzing sub-clinical Kaiso ${ }^{T g}$ mice. Thus, we first analysed the small intestine of 3-mo. old mice for signs of chronic inflammation. The small intestines of 3-mo. old Kaiso ${ }^{T g}$ mice do not exhibit signs of widespread chronic intestinal inflammation visible in 12 -mo. old Kaiso ${ }^{T g}$ mice - i.e. extensive villus blunting, thickened submucosa, extensive neutrophil infiltration, etc. [19] (Fig. 1a). Consistent with the lack of inflammation-related tissue damage, there was no 

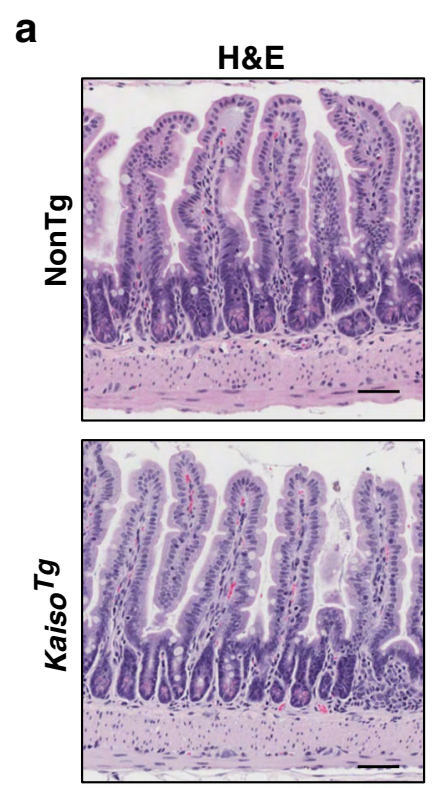

b

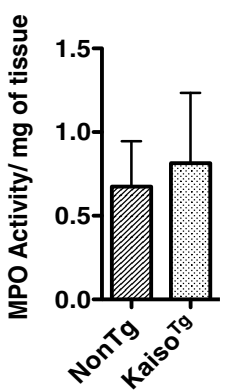

C

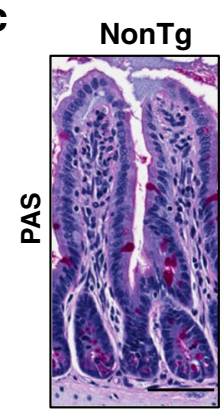

d
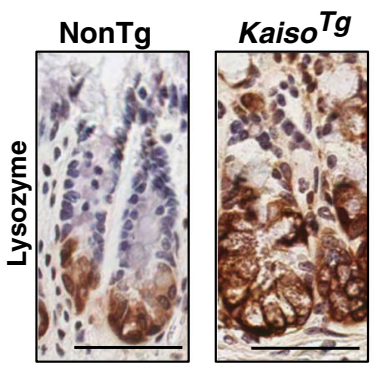

e

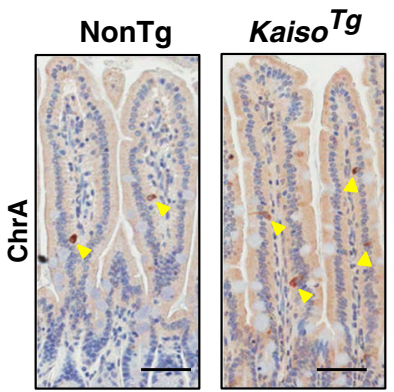

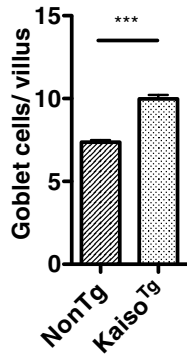
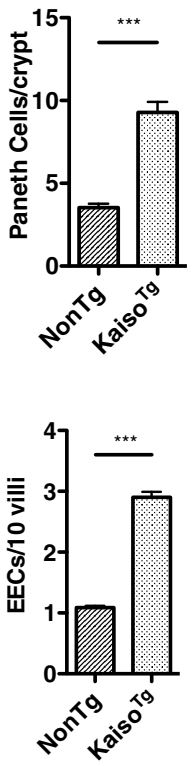

Fig. 1 Kaiso phenocopies loss of canonical Notch signaling independently of intestinal inflammation. a Hematoxylin \& eosin (H\&E) staining of 3-month (mo.) old Kaiso ${ }^{T g}$ intestines do not show widespread intestinal tissue damage due to chronic inflammation. b Myeloperoxidase (MPO) activity of 3-mo. old Kaiso ${ }^{T g}$ is unchanged relative to age-matched NonTg mice. c-e 3-mo. old Kaiso ${ }^{T g}$ mice exhibit an increase goblet (PAS - periodic acid Schiff), Paneth (Lysozyme) and enteroendocrine (EEC; ChrA - chromogranin A, yellow arrowheads) cell numbers relative to age-matched NonTg mice. Statistical significance determined using student's t-test. Error bars are SEM, ${ }^{* * *} p<0.0005$. Scale bar, $50 \mu \mathrm{m}$

significant difference in myeloperoxidase (MPO) activity (a measure of activated neutrophils and a surrogate marker for inflammation) between 3-mo. old NonTg and Kaiso $^{T g}$ mice ( $p=0.792$; Fig. $\left.1 \mathrm{~b}\right)$.

Since we did not observe neutrophil-specific intestinal inflammation in 3-mo. old Kaiso ${ }^{T g}$ mice, we next assessed the secretory cell types in these mice. Small intestines were stained with periodic acid-Schiff stain (PAS), or labeled with antibodies against lysozyme and chromogranin A to identify goblet, Paneth and enteroendocrine cells, respectively. We observed a significant increase in all three types of secretory cells $(p<0.0001)$ in 3-mo. old Kaiso ${ }^{T g}$ mice compared to NonTg siblings (Fig. 1c, d and e).

Given that Kaiso overexpression drives an increase in secretory cells, we hypothesized that loss of Kaiso would result in a reduced number of secretory cells. We thus examined age-matched $\mathrm{Kaiso}^{-/ y}$ and NonTg mice for goblet cell numbers using alcian blue. Intriguingly, we did not observe a significant difference in the number of goblet cells (Additional file 1: Fig. S1).

Several studies have highlighted the critical role of Notch signaling in mediating intestinal cell fate decisions, by demonstrating that loss of Notch signaling produces an increase in secretory cell types [1, 8-13, 18, 36, 37]. Thus, we examined Kaiso ${ }^{T g}$ intestinal tissues for the expression levels of two downstream Notch pathway effectors, the Hes1 and Hes5 transcription factors, which are known to inhibit secretory cell fates [13]. Indeed, we observed reduced Hes 1 and Hes 5 expression in Kai$s o^{T g}$ compared to NonTg mice as determined by IHC 


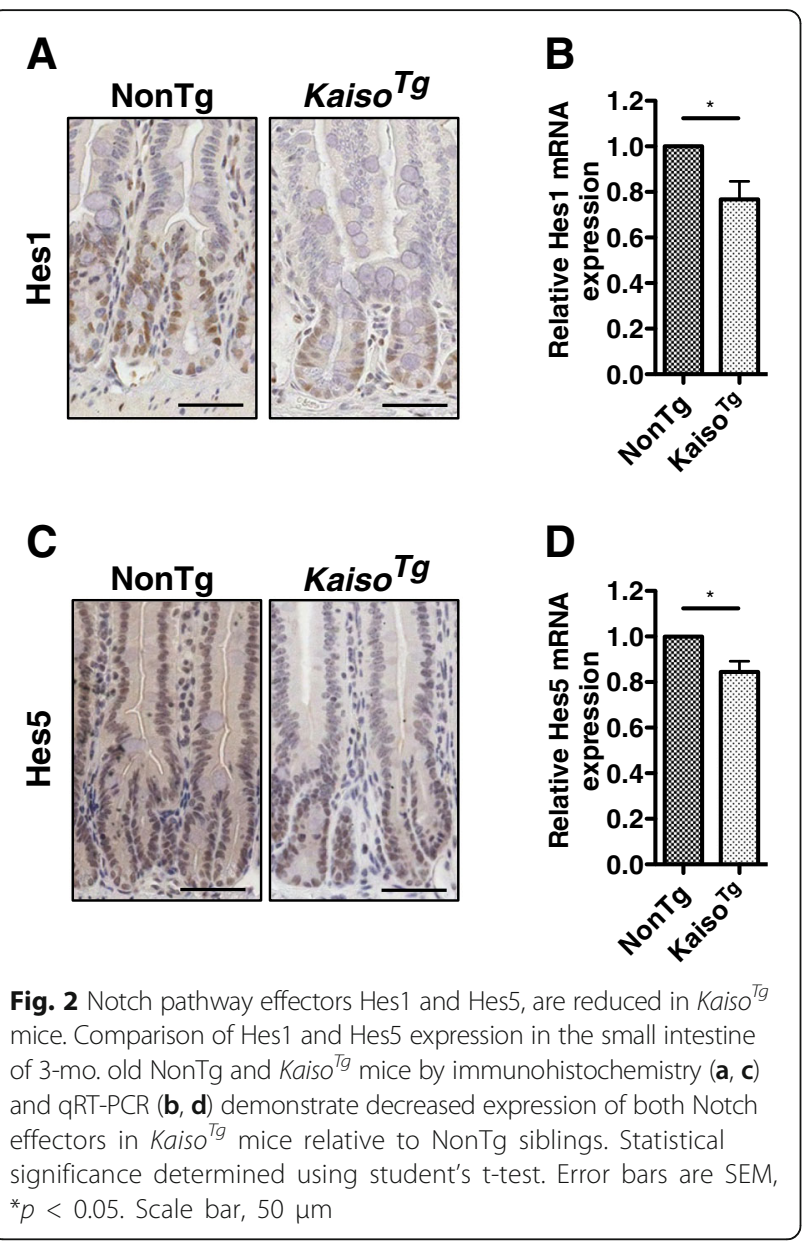

(Fig. 2a, c). Examination of Hes1 and Hes5 transcript levels using mRNA isolated from intestinal epithelial cells (IECs) revealed marginal, but significant, reductions in the relative expression levels of both Hes 1 and Hes5 in Kaiso ${ }^{T g}$ mice compared to NonTg siblings (Fig. 2b, d). Together, these data suggest that Kaiso overexpression inhibits, but does not completely abolish, Notch pathway activation in intestinal cells. More importantly, our data indicate that Kaiso's effects on Notch signaling precede the onset of Kaisoinduced intestinal inflammation.

\section{Kaiso inhibits Notch1 but not Notch2 expression in the small intestine.}

Since our data suggest that Kaiso overexpression suppresses Notch signaling in the intestine of 3-mo. old mice, we next sought to determine how Kaiso regulates this pathway. Although all four Notch receptors are expressed in the intestinal mucosa, Notch1 and Notch2 are specifically expressed in IECs and are responsible for governing cell fate decisions in the intestines [11, 36, 38, 39]. IECs from 3-mo. old Kaiso ${ }^{T g}$ and NonTg mice were isolated and assayed for Notch1 and Notch 2 mRNA levels. We observed a significant reduction in Notch1 mRNA expression in Kaiso ${ }^{T g}$ compared to NonTg mice ( $p=0.014$, Fig. 3a). While Notch 2 mRNA levels were also reduced, the change was not statistically significant $(p=0.072$, Fig. 3a).

Notch pathway activation is characterized by the cytoplasmic release and nuclear translocation of the NICD [40]. Since we observed a reduction in Notch1 mRNA expression levels, we next assessed Notch1-ICD (N1ICD) levels in Kaiso $^{T g}$ mice by examining the tissue distribution of N1-ICD in Kaiso ${ }^{T g}$ mice using IHC. As expected, N1-ICD was restricted to the nuclei of crypt epithelial cells in both NonTg and Kaiso ${ }^{T g}$ mice (Fig. 3b). However, Kaiso ${ }^{T g}$ intestinal tissues exhibited an overall reduction in N1-ICD expression compared to their NonTg counterpart (Fig. 3b). To further validate our observations, we examined N1-ICD expression in three stable Kaiso-depleted colon cancer cell lines, as we postulated that loss of Kaiso would result in increased N1-ICD expression. Indeed, N1-ICD expression was increased in stable Kaiso-depleted (shKaiso) SW480, HCT116 and HT29 cells compared to control cell lines that stably express a scrambled shKaiso sequence (shScr) (Fig. 3c).

To examine Kaiso's effects on N1-ICD function, we performed artificial promoter-reporter luciferase assays using parental HCT116 and SW480 colon cancer cells. HT29 cells were omitted from this analysis since these cells exhibited poorer transfection efficiency compared to HCT116 and SW480 cells. A Gaussia luciferase vector containing 4 tandem copies of the RBP-J/CSL consensus sequence (pGLuc-4XCSL) was co-transfected with expression vectors encoding N1-ICD (pCAGGS-N1-ICD) and Kaiso (pcDNA3-hKaiso). Co-expression of pCAGGS-N1ICD and pGLuc-4XCSL resulted in a $\sim 15$ - and $\sim 18$-fold increase in pGLuc-4XCSL activity in HCT116 and SW480 cells, respectively ( $p<0.0001$ ) (Fig. 3d). However, coexpression with pcDNA3-hKaiso attenuated N1-ICDmediated transactivation of pGLuc-4XCSL in both cell lines, in a dose-dependent manner $(p<0.0001)$ (Fig. 3d). Collectively, these data demonstrate that Kaiso inhibits Notch1 signaling in intestinal cells.

Dll-1 expression is reduced in Kaiso ${ }^{\mathrm{Tg}}$ mice.

Previous reports have shown that Notch1 and Notch2 act redundantly in the intestine; thus the single loss of either receptor is insufficient to attenuate Notch signaling [11]. Hence, we surmised that the secretory cell phenotype observed in Kaiso ${ }^{T g}$ mice (Fig. 1) could not be attributed to a reduction in Notch1 expression alone, and that Kaiso may be inducing the secretory cell phenotype via regulation of other components of the Notch pathway. Since Dll-1 and Dll-4 are also implicated in cell fate decisions in the intestine [8], we interrogated 


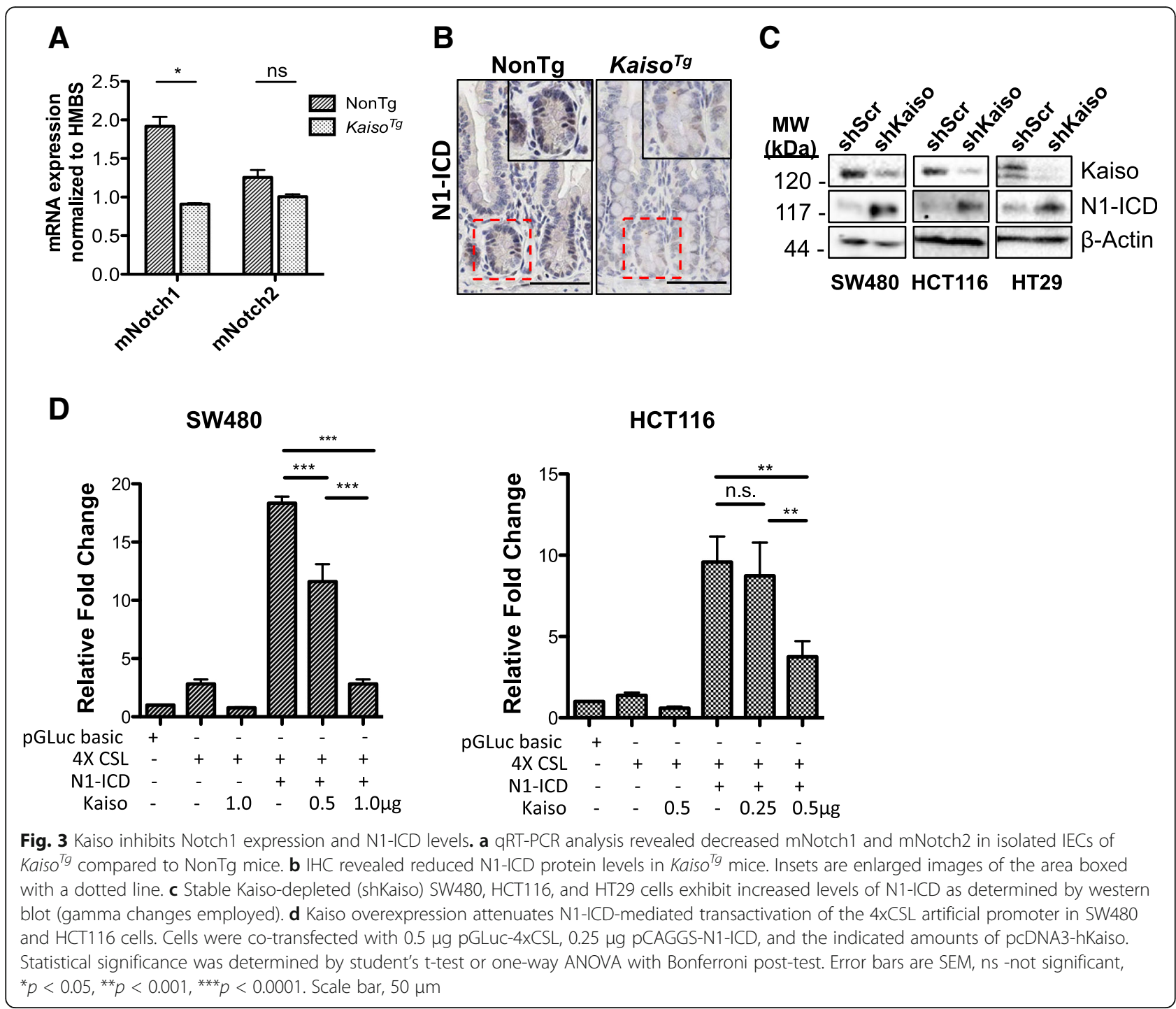

the effects of Kaiso overexpression on Dll-1 and Dll-4 expression in our Kaiso ${ }^{T g}$ mice. We first quantified $D l l-1$ and Dll-4 transcript levels by qRT-PCR analysis of harvested IECs from 3-mo. old NonTg and Kaiso ${ }^{T g}$ mice. Interestingly, we observed an $\sim 2$-fold reduction in Dll-1 transcript levels in Kaiso $^{T g}$ mice compared to NonTg siblings ( $p=0.04)$, while $\mathrm{Dll}-4$ transcript levels remained relatively unchanged $(p=0.303)$ (Fig. 4a). We next assessed the in vivo expression levels of Dll-1 and Dll-4 in our Kaiso ${ }^{T g}$ intestines by IHC and found a striking reduction in Dll-1 positive cells in intestines from Kaiso ${ }^{T g}$ mice compared to NonTg siblings (Fig. 4b). Consistent with qRT-PCR analyses, Dll-4 tissue expression was relatively unchanged (Fig. $4 \mathrm{~b})$. In support of our findings, western blot analysis also revealed a marked reduction in Dll-1 protein levels in isolated Kaiso ${ }^{T g}$ IECs relative to NonTg (Fig. 4c).

To further validate our in vivo findings, Dll-1 transcript and protein levels were also assessed in stable
Kaiso-depleted SW480, HCT116 and HT29 cells. Increased levels of Dll-1 transcripts were observed in all three Kaiso-depleted cell lines (SW480, HCT116 and HT29) relative to the scrambled controls, but the change in Dll-1 mRNA levels was only statistically significant in Kaiso-depleted HT29 cells $(p=0.012)$ (Fig. 4d). Immunoblot analysis of Dll-1 protein expression revealed an $\sim 1.2-, \quad \sim 1.5-$ and $\sim 2.6$-fold increase in Kaiso-depleted SW480, HCT116 cells, and HT29 cells, respectively (Fig. 4e). Together, these data demonstrate that Kaiso inhibits Dll-1 expression in intestinal cells, and raise the possibility that the observed increase in secretory cell types is due to Kaiso-mediated Dll-1 loss.

Kaiso associates endogenously with the DLL1 but not the NOTCH1 promoter.

Our group and others have shown that Kaiso possesses dual-specificity DNA-binding abilities, and recognizes 


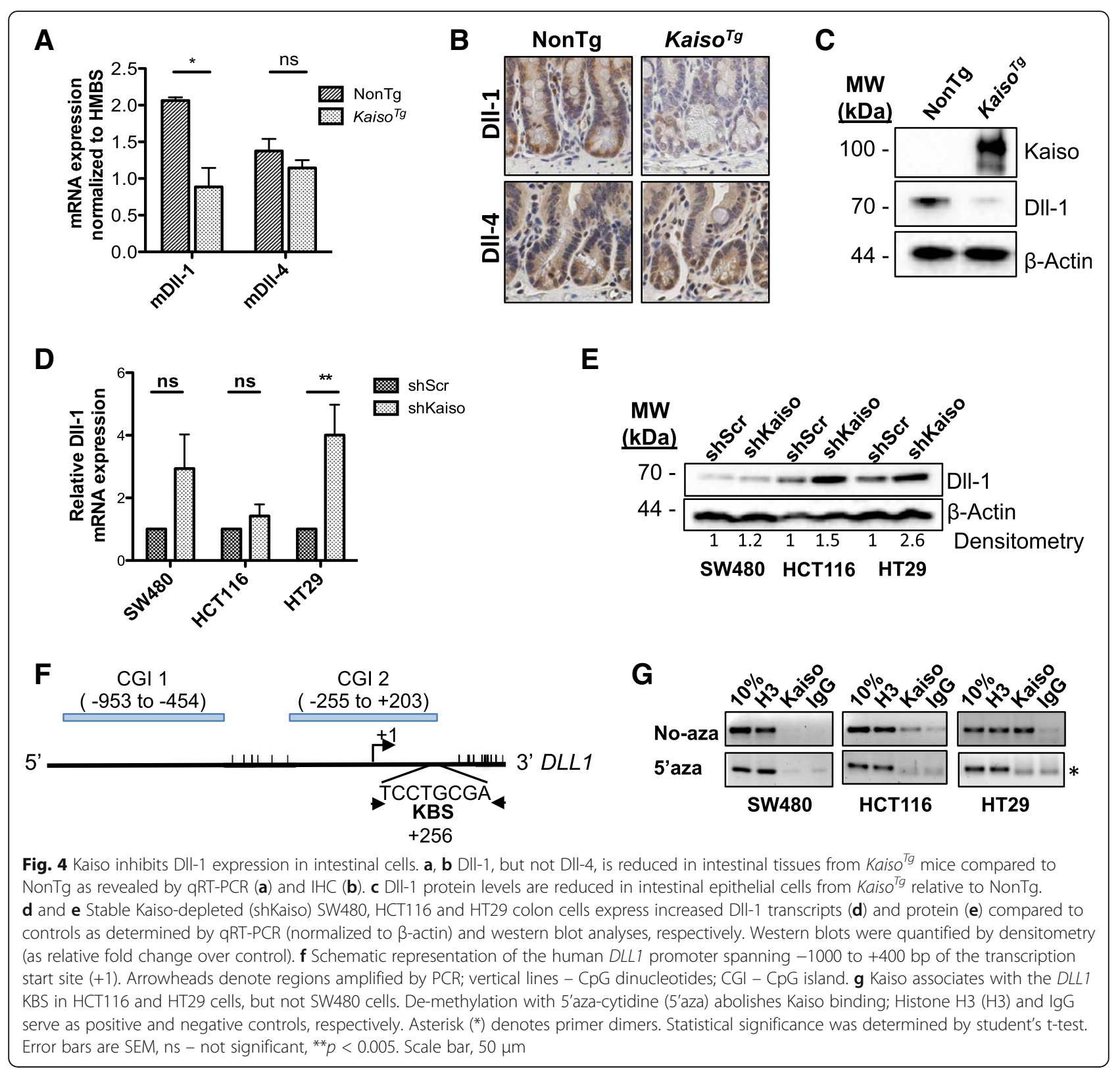

and binds both the sequence-specific Kaiso binding site (KBS - TCCTGCNA, core sequence underlined) and methylated CpG dinucleotides [25, 41-44]. Given Kaiso's effects on the expression of Dll-1 and Notch1 in intestinal cells, we next assessed whether either of these Notch pathway components might be putative Kaiso target genes. Examination of the DLL1 and NOTCH1 gene promoters revealed the presence of one or several putative KBS sequences, and numerous CpG dinucleotides. We assayed whether Kaiso associated with these loci in a sequence- or methylation-specific manner. To this end, chromatin was isolated from untreated SW480, HCT116 and HT29 cells, and from cells treated with 5 4 M 5'-aza-cytidine for 5 consecutive days. Chromatin immunoprecipitation (ChIP) was performed using the Kaiso-specific 6F monoclonal antibody [28], or with histone $\mathrm{H} 3$ and non-specific IgG as positive and negative controls, respectively. The DLL1 gene promoter spanning -1000 to $+400 \mathrm{bp}$ of the transcriptional start site (TSS) contains 2 CpG islands (CGIs; Fig. 4f \& Additional file 2: Fig. S2), and has one full KBS located $256 \mathrm{bp}$ downstream of the TSS which is not only surrounded by 6 CpG dinucleotides, but also itself encompasses a CpG dinucleotide, TCCTGCGA (Fig. 4f). Thus, ChIP-PCR was performed on the region encompassing the KBS and surrounding $6 \mathrm{CpG}$ dinucleotides to ascertain whether Kaiso binding occurred in a sequence-specific or methylation-dependent manner. ChIP analysis revealed 
that Kaiso associated endogenously with the DLL1 KBS site in untreated HCT116 and HT29 cells, but not in SW480 cells (Fig. 4g). However, Kaiso binding was abolished upon de-methylation with 5 '-aza-cytidine (Fig. 4g, 5'aza-treated), suggesting that Kaiso associates with the DLL1 promoter in a methylation-dependent manner despite the presence of the KBS.

The NOTCH1 promoter contains three core KBS sites at positions $-1599 \mathrm{bp},-1464 \mathrm{bp}$ and $-341 \mathrm{bp}$ of the TSS. However, endogenous Kaiso binding was not observed at any of these loci in the colon cancer cell lines tested (data not shown), despite the presence of multiple CPG dinucleotides in the region analyzed.

Kaiso promotes expression of the Notch ligand Jagged-1. Unlike Dll-1 and Dll-4, the Notch ligand Jagged-1 is dispensable for determining secretory cell fate decisions in the intestines [8]. However mounting evidence has highlighted an important role for Jagged-1 in Notchmediated colon carcinogenesis and progression [20-23, 45-47]. Given that Kaiso promotes polyp formation in the $\mathrm{Apc}^{\mathrm{Min} /+}$ mouse model of colon cancer and reduces overall survival of $A p c^{\mathrm{Min} /+}$ mice $[24,26]$, we were prompted to examine Jagged-1 expression and tissue localization in our Kaiso ${ }^{T g}$ mice. Using immunofluorescence staining, we observed Jagged-1 positive cells primarily in the crypts of both NonTg and Kaiso ${ }^{T g}$ mice (Fig. 5a). Notably, Kaiso-overexpression led to enhanced Jagged-1 expression, and an increase in the number of positively stained cells compared to NonTg counterparts (Fig. 5a). Consistent with this finding, loss of Kaiso in stable Kaiso-depleted SW480 cells resulted in reduced Jagged-1 transcript and protein levels compared to control cells (Fig. 5b, c). Notably, HCT116 and HT29 cells do not express significant levels of Jagged-1 mRNA or protein, and thus the effects of Kaiso depletion in these cell lines were negligible (data not shown). Collectively, these data show that, unlike its effects on Notch1 and Dll-1 expression, Kaiso promotes Jagged-1 expression in intestinal cells.

Since we observed a positive correlation between Jagged-1 and Kaiso expression in our studies, and Kaiso has also been found to activate expression of some target genes [48-50], we investigated whether JAG1 might also be a putative Kaiso target. We searched a region spanning -1000 to $+2500 \mathrm{bp}$ of the TSS for putative KBS sites and identified at least five core KBS sites. However we focused on the $-200 \mathrm{bp}$ to $+2500 \mathrm{bp}$ region since this region had a high frequency of putative KBS sites, and also showed binding at 2 loci on the UCSC ENCODE Database [51]. Five putative CGIs and four core KBS sites (i.e. CTGCNA) were identified in the -200 to +2500 region of the JAG1 promoter at positions $-6 \mathrm{bp}$ (KBS-A), +2017 bp (KBS-B), +2188 bp and +2251 bp

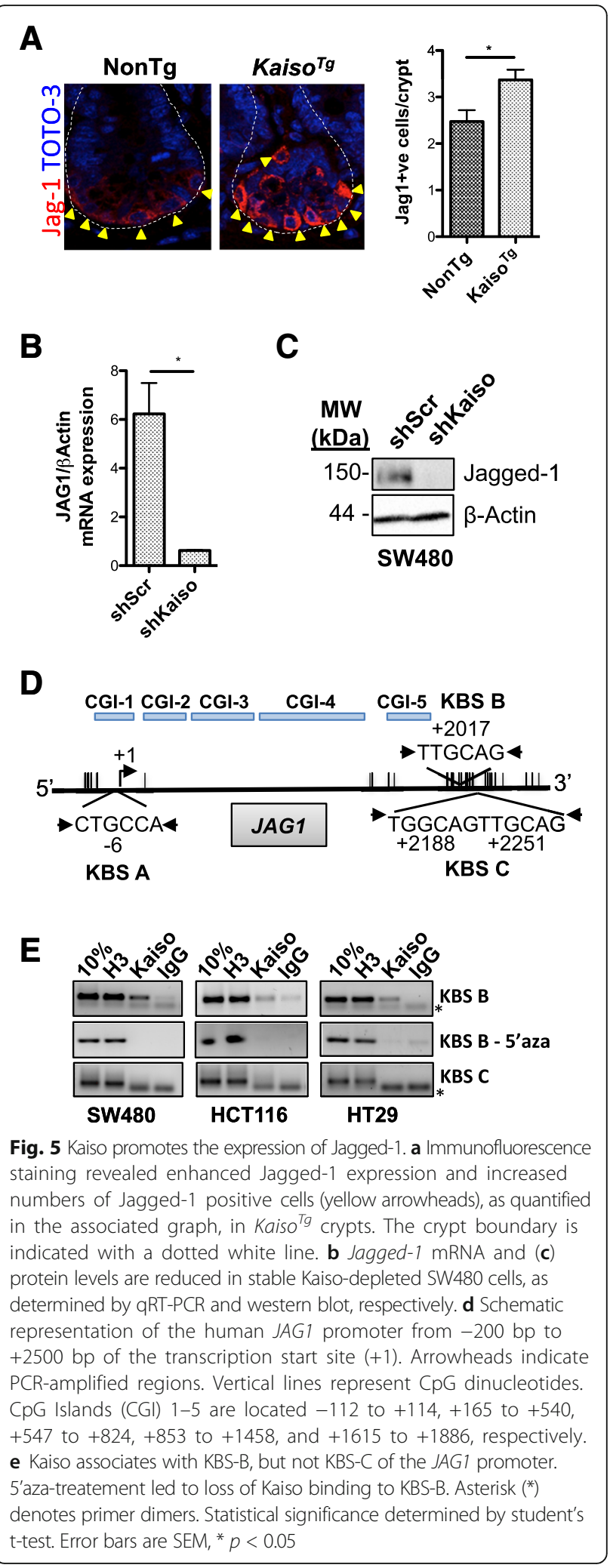

(collectively, KBS-C) relative to the TSS (Additional file 3: Fig. S3, Fig 5d). Primers were designed to PCR amplify each locus, however given the proximity of 
the +2188 and $+2251 \mathrm{KBSs}$ to each other, primers were designed to encompass both KBSs. Kaiso associated weakly with KBS-A in SW480 and HCT116 cells, but not in HT29 cells (data not shown), and no association between Kaiso and KBS-C was detected in any of the cell lines tested (Fig. 5e). However, strong endogenous Kaiso binding was detected at the KBS-B locus in all three cell lines, which was abrogated upon de-methylation with 5'aza-cytidine (Fig. 5e). Notably, KBS-B and -C both occur outside the CGIs but are both surrounded by several CpG dinucleotides. Thus similar to the DLL1 KBS, Kaiso associates with the JAG1 KBS-B locus in a methylationdependent manner.

In summary, our data demonstrate that Kaisomediated regulation of Notch signaling in intestinal tissues occurs via regulation of multiple Notch pathway components. Indeed, our finding that Kaiso associates with the DLL1 and JAG1 promoters in a methylationdependent manner implicates them as putative Kaiso target genes, and suggests that this may be one mechanism by which Kaiso regulates Notch signaling in intestinal cells. Remarkably, Kaiso appears to repress Dll-1 expression, which plays an important role in intestinal cell differentiation, but activates Jagged-1 expression, which is dispensable for cell fate determination.

\section{Discussion}

The highly conserved Notch signaling pathway is fundamental for several biological processes, including binary cell fate decisions in the intestine where it functions to inhibit secretory cell differentiation, while promoting the absorptive cell fate, reviewed in $[1,2,5]$. Herein we demonstrate that the POZ-ZF transcription factor Kaiso, whose overexpression enhances intestinal polyp formation in $\mathrm{Apc}^{\mathrm{Min} / \mathrm{+}}$ mice [26] and drives spontaneous intestinal inflammation, regulates the Notch pathway and secretory cell fates in intestinal tissues [19].

During our initial characterization of 12-mo. old Kaiso $^{T g}$ mice, we noticed that Kaiso $^{T g}$ mice exhibited an increase in neutrophil-specific intestinal inflammation [19] (and our unpublished data) and a Notch-depletion phenotype (i.e. increased numbers of secretory cell types). By examining sub-clinical 3-mo. old mice in this study, we found that younger Kaiso ${ }^{T g}$ mice do not display widespread signs of chronic inflammation, but still phenocopy loss of Notch signaling. This finding suggests that the Notch-depletion phenotype in Kaiso ${ }^{T g}$ mice precedes intestinal inflammation (Fig. 1) and may in fact play a role in the pathology of Kaiso-induced chronic inflammation at later ages. Similar findings were observed by Obata et al., who demonstrated that Notch-signaling inhibition via IEC-specific deletion of Rbpj (RBP-J ${ }^{I E C / \Delta}$ ) resulted in spontaneous inflammation in the colons of these mice [52]. Similar to Kaiso ${ }^{T g}$, RBP-I IEC/ ${ }^{I}$ mice exhibited increased numbers of secretory cells throughout the intestine, as well as augmented neutrophil infiltration in inflamed regions [52].

Notch pathway activation culminates in the expression of the Hes transcription factor family, which function to suppress the secretory cell lineage in the intestinal epithelium [13, 38]. While Hes1-deficiency is sufficient to drive increased secretory cell numbers in immature mice, this effect is counteracted by Hes 3 and Hes 5 expression after 2 months of age [13]. Indeed, combined loss of Hes $1 / 3 / 5$ resulted in augmented secretory cell numbers compared to loss of Hes1 alone in 2-mo. old mice [13]. Thus, our hypothesis that Kaiso promotes secretory cell differentiation via Notch pathway inhibition is further strengthened by the observation that 3mo. old Kaiso ${ }^{T g}$ mice exhibit reduced expression of both Hes 1 and Hes5 compared to age-matched NonTg siblings (Fig 2). However, qRT-PCR analyses revealed only a modest reduction in mRNA levels of both transcription factors, suggesting that Notch signaling is not completely abolished in Kaiso ${ }^{T g}$ epithelium. This possibility is consistent with our observation that only Dll-1 but not Dll-4, is reduced in Kaiso ${ }^{T g}$ mice.

Using tamoxifen-inducible mouse models, Pellegrinet et al. demonstrated that $D l l-1$ ablation was sufficient to cause a mild increase in goblet cells, while loss of $D l l-4$ alone did not affect goblet cell numbers [8]. This study was subsequently substantiated by Stamataki and colleagues, who showed that inducible Dll-1 knock-out led to an increase in enteroendocrine and Paneth cell numbers [18]. Notably however, genetic loss of both $\mathrm{Dll}-1$ and $D l l-4$ produces a more striking phenotype, where the intestinal epithelium undergoes post-mitotic goblet cell conversion [8]. Moreover, while Dll-1 is able to fully rescue loss of Dll-4, Dll-4 is only partially able to rescue loss of Dll-1. Thus, the finding that Dll-1, and not Dll-4, is reduced in Kaiso ${ }^{T g}$ mice compared to their NonTg siblings (Fig. 4), is consistent with these previous reports $[8,18]$, and may explain why 3 -mo. old mice exhibit a relatively modest secretory cell phenotype compared to other mouse models of Notch inhibition [8, 11, 53, 54]. Moreover, although we also observed a reduction in Notch1 expression (Fig. 3), the functional redundancy of Notch1 and Notch2 in the intestine [11] suggests that the Notch-depletion phenotype caused by constitutive Kaiso overexpression is due to either a reduction in Dll1 alone [8], or a combination of both Notch-1 and Dll-1 inhibition. Nevertheless, our findings support a role for Kaiso in secretory cell fate decisions through suppression of Notch signaling

Although Jagged-1 is dispensable for cell differentiation in the intestines [8], increasing evidence supports a role for Jagged-1 in colon cancer [20, 22, 23, 45, 46]. While the enhanced Jagged-1 positivity in Kaiso ${ }^{T g}$ mice 
was unexpected (Fig. 5), the data is consistent with our previous findings that Kaiso potentiates $\mathrm{Apc}^{\mathrm{Min} / \mathrm{+}}$-mediated tumorigenesis $[24,26]$, and raises the possibility that one mechanism by which Kaiso promotes intestinal tumourigenesis is via Jagged-1 activation.

Several previous studies have demonstrated that Kaiso is capable of binding to DNA using various mechanisms: either in a sequence-specific manner (via the KBS), via methyl-CpG dinucleotides, or a combination of both $[25,27,41,42,49,55,56]$. The observation that Kaiso's association with the DLL1 and $J A G 1$ promoters is abolished upon demethylation with 5 '-aza-cytidine suggests that Kaiso primarily binds to these regions via methyl-CpG, and not KBS-specific, binding mechanisms. While extensive characterization of Kaiso's binding mechanisms to the DLL1 and JAG1 promoters is beyond the scope of this study, it is possible that the presence of a KBS may act to increase the specificity of Kaiso's association with methylCpGs. Indeed, we reported such a phenomenon with the CCND1 promoter, where Kaiso was found to associate with the $+69 \mathrm{KBS}$ in a methylation-dependent manner. Notably however, mutation of the core KBS nucleotides surrounding the methyl-CpG weakened Kaiso's association with the CCND1 + 69 KBS site, suggesting that the presence of the $\mathrm{KBS}$ acts to strengthen and/or increase the specificity of binding to methyl-CpG sites [25]. Intriguingly, Kaiso did not associate with JAG1 KBS-C or the NOTCH1 KBS loci despite the presence of surrounding CpG dinucleotides, a phenomenon that underscores the complexity of Kaiso-mediated transcriptional regulation of its target genes.

While Kaiso overexpression was found to promote the formation of secretory cell types, contrary to our hypothesis, we did not observe a significant change in the number of goblet cells in $\mathrm{Kaiso}^{-/ y}$ mice (Additional file 1: Fig. S1). During their characterization of Kaiso $^{-/ y}$ mice, Prokhortchouk and colleagues also reported that loss of Kaiso did not produce gross morphological defects, nor did it cause significant alterations to Kaiso-target gene expression [24]. Given that the Kaiso-like proteins, ZBTB4 and ZBTB38 also bind DNA sequences similar to the KBS [43, 57], it is possible that the lack of a secretory cell phenotype in the $\mathrm{Kaiso}^{-/ y}$ mice is due to functional redundancy conferred by these proteins, though this remains to be determined empirically. Nevertheless, the finding that Kaiso overexpression exerts distinct effects on Notch1, Dll-1 and Jagged-1 may still be physiologically relevant, since Kaiso expression is elevated in several diseases, including breast [49, 58], prostate [59], and colon cancer [24, 26], and in some cases of Crohn's disease (our unpublished data).

\section{Conclusion}

In conclusion, this study describes at least one mechanism by which Kaiso regulates Notch-mediated intestinal homeostasis. Specifically, we demonstrate that Kaiso inhibits the expression of Notch1 and the Notch ligand Dll-1, but enhances the expression of Jagged-1. We postulate that Kaiso-mediated repression of Dll-1 is sufficient to promote the increase in secretory cells observed in our mice. While Kaiso-mediated activation of Jagged1 in the intestinal epithelium likely does not contribute to the secretory cell phenotype, it is possible that the Kaiso/Jagged-1 interaction contributes to colon cancer progression.

\section{Additional files}

\begin{abstract}
Additional file 1: Figure S1. Kaiso ${ }^{-1 y}$ mice do not exhibit a goblet cell defect. (A) Immunofluorescence staining of Kaiso ${ }^{-1 y}$ mice confirm Kaisodepletion in the intestinal epithelium. Intestines were counterstained with DAPI (4, 6-diamidino-2-phenylindole) to label the nuclei. (B) Goblet cells from three 4-week old NonTg and Kaiso ${ }^{-1 y}$ mice were labeled with alcian blue and quantified. Kaiso ${ }^{-1 y}$ mice do not exhibit a significant change in goblet cells, as determined by student's t-test. (PDF 770 kb)
\end{abstract}

Additional file 2: Figure S2. CpG island prediction of the $D L L 1$ promoter. The DLL1 promoter spanning -1000 to +400 bp of the TSS was analyzed for putative CpG islands. This region is GC-rich and contains two potential CpG islands at -953 to $-454 \mathrm{bp}$ and -255 to $+203 \mathrm{bp}$ of the TSS (+1), respectively. (PDF $73 \mathrm{~kb}$ )

Additional file 3: Figure S3. CpG island prediction of the minimal JAG1 promoter. The JAG1 gene spanning -200 to +2500 bp of the TSS was analyzed for putative $\mathrm{CpG}$ islands. This GC-rich region harbors 5 potential CpG islands. (PDF $51 \mathrm{~kb}$ )

\begin{abstract}
Abbreviations
5'-aza: 5' aza-cytidine; ANOVA: Analysis of variance; CGl: CpG Island; ChIP: Chromatin immunoprecipitation; CSL: CBF1, suppressor of hairless, Lag2; DAPI: 4, 6- Diamidino-2-phenylindole; DII: Delta like ligand; DSL: Delta/ Serrate/Lag2; Hes: Hairy enhancer of split; H \& E: Hematoxylin and eosin; HMBS: Hydroxymethylbilane synthase; HTAB: hexadecyltrimethylammonium bromide; IEC: Intestinal epithelial cells; IHC: Immunohistochemistry; Kaiso $^{\text {Tg }}$ : Kaiso transgenic; KBS: Kaiso binding site; LSB: Laemmli sample buffer; Mo.: Month; MPO: Myeloperoxidase; N1-ICD: Notch1 intracellular domain; NICD: Notch intracellular domain; NonTg: Non-transgenic; PAS: Periodic acid Schiff; PBS: Phosphate buffered saline; POZ-ZF: Pox virus and zinc finger zinc finger; qRT-PCR: Quantitative real time polymerase chain reaction; RBP$\mathrm{J}$ : Recombination signal binding protein for immunoglobulin kappa J region; SEM: Standard error of the mean; TBS: Tris buffered saline; TSS: Transcription start site
\end{abstract}

\section{Acknowledgements}

The authors gratefully acknowledge Dr. Albert Reynolds for providing the Kaiso rabbit polyclonal antibody used in this study. We would also like to acknowledge Shawn Hercules and Blessing Bassey-Archibong for their critical revision of this manuscript, and Shatha Jaber for her technical assistance.

\section{Availability of data and materials}

Data generated during this study are included in this published article, and are available from the corresponding author on reasonable request.

\section{Funding}

JMD was funded by the Canadian Institutes of Health Research MOP-84320 and an NSERC Discovery Grant \# RGPIN6442-15. 


\section{Authors' contributions}

SCR and JMD conceptualized the study. KK assisted with ChIP and IHC experiments; CCP performed the MPO assay and assisted with processing of Kaiso ${ }^{T g}$ intestinal tissues used for $\mathrm{IHC}$; AA assisted with $\mathrm{IHC}$ and with blind quantification of cells; SZ and EP provided IF and alcian blue staining of Kaiso $^{-1 y}$ intestinal tissues. SCR performed all other experiments, analyzed and interpreted the data, and wrote the manuscript along with JMD. All authors read and approved the final manuscript.

\section{Competing interests}

The authors declare that they have no competing interests.

\section{Consent for publication}

Not applicable

\section{Ethics approval and consent to participate}

All mouse-handling protocols were performed in accordance with the McMaster Animal Research Ethics Board under the Animal Utilization Protocol \#14-08-29.

\section{Author details}

'Department of Biology, McMaster University, Hamilton L8S 4K1, ON, Canada. ${ }^{2}$ Current address: Department of Biochemistry and Biomedical Sciences, Michael G. DeGroote Institute for Infectious Disease Research, McMaster University, Hamilton L8N 3Z5, ON, Canada. ${ }^{3}$ Current address: Department of Life Science, University of the West Indies at St. Augustine, St. Augustine, Trinidad and Tobago. ${ }^{4}$ Federal Research Centre of Biotechnology, Russian Academy of Sciences, Moscow, Russian Federation117312.

Received: 30 December 2016 Accepted: 5 June 2017

Published online: 21 June 2017

\section{References}

1. de Santa Barbara P, Van den brink GR, Roberts DJ. Development and differentiation of the intestinal epithelium. Cell Mol Life Sci. 2003;60(7): 1322-32.

2. Crosnier C, Stamataki D, Lewis J. Organizing cell renewal in the intestine: stem cells, signals and combinatorial control. Nat Rev Genet. 2006;7(5):349-59.

3. Gassler N, et al. Molecular characterisation of non-absorptive and absorptive enterocytes in human small intestine. Gut. 2006;55(8):1084-9.

4. Barker N. Adult intestinal stem cells: critical drivers of epithelial homeostasis and regeneration. Nat Rev Mol Cell Biol. 2014;15(1):19-33.

5. Noah TK, Donahue B, Shroyer NF. Intestinal development and differentiation. Exp Cell Res. 2011;317(19):2702-10.

6. Umar S. Intestinal Stem Cells. Curr Gastroenterol Rep. 2010;12(5):340-8.

7. Scoville DH, et al. Current View: Intestinal Stem Cells and Signaling. Gastroenterology. 2008;134(3):849-64.

8. Pellegrinet $L$, et al. DII1- and D\|4-Mediated notch signaling are required for homeostasis of intestinal stem cells. Gastroenterology. 2011;140(4): 1230-40. e1-7.

9. Yang Q, et al. Requirement of Math1 for secretory cell lineage commitment in the mouse intestine. Science. 2001;294(5549):2155-8.

10. Hansson EM, Lendahl U, Chapman G. Notch signaling in development and disease. Semin Cancer Biol. 2004;14(5):320-8.

11. Riccio $\mathrm{O}$, et al. Loss of intestinal crypt progenitor cells owing to inactivation of both Notch1 and Notch2 is accompanied by derepression of CDK inhibitors p27Kip1 and p57Kip2. EMBO Rep. 2008:9(4):377-83.

12. Okamoto $R$, et al. Requirement of Notch activation during regeneration of the intestinal epithelia. Am J Physiol Gastrointest Liver Physiol. 2009;296(1):G23-35

13. Ueo T, et al. The role of Hes genes in intestinal development, homeostasis and tumor formation. Development. 2012;139(6):1071-82.

14. Kageyama R, Ohtsuka T, Kobayashi T. The Hes gene family: repressors and oscillators that orchestrate embryogenesis. Development. 2007;134(7):1243-51.

15. Kopan R, llagan MX. The canonical Notch signaling pathway: unfolding the activation mechanism. Cell. 2009;137(2):216-33.

16. Fleming RJ. Structural conservation of Notch receptors and ligands. Semin Cell Dev Biol. 1998;9(6):599-607.
17. Demitrack ES, Samuelson LC. Notch regulation of gastrointestinal stem cells. J Physiol. 2016:594(17):4791-803.

18. Stamataki D, et al. Delta1 expression, cell cycle exit, and commitment to a specific secretory fate coincide within a few hours in the mouse intestinal stem cell system. PLoS One. 2011;6(9):e24484.

19. Chaudhary $R$, et al. The POZ-ZF Transcription Factor Kaiso (ZBTB33) induces inflammation and progenitor cell differentiation in the murine intestine. PLoS One. 2013;8(9):e74160.

20. Rodilla $V$, et al. Jagged1 is the pathological link between Wnt and Notch pathways in colorectal cancer. Proc Natl Acad Sci U S A. 2009; 106(15):6315-20.

21. Guilmeau S, et al. Heterogeneity of Jagged1 expression in human and mouse intestinal tumors: implications for targeting Notch signaling. Oncogene. 2010;29(7):992-1002.

22. Dai $Y$, et al. Silencing of Jagged 1 inhibits cell growth and invasion in colorectal cancer. Cell Death Dis. 2014;5:e1170.

23. Sugiyama $M$, et al. High expression of the Notch ligand Jagged-1 is associated with poor prognosis after surgery for colorectal cancer. Cancer Science. 2016.

24. Prokhortchouk A, et al. Kaiso-deficient mice show resistance to intestinal cancer. Mol Cell Biol. 2006;26(1):199-208.

25. Donaldson NS, et al. Kaiso represses the cell cycle gene cyclin D1 via sequence-specific and methyl-CpG-dependent mechanisms. PLoS One. 2012;7(11):e50398.

26. Pierre CC, et al. Kaiso overexpression promotes intestinal inflammation and potentiates intestinal tumorigenesis in $\mathrm{Apc}^{\mathrm{Min} /+}$ mice. BBA- Mol Basis Dis. 2015;1852(9):1846-55.

27. Pierre CC, et al. Methylation-dependent regulation of hypoxia inducible factor-1 alpha gene expression by the transcription factor Kaiso. BBA - Gene Regul Mech. 2015;1849(12):1432-41.

28. Daniel JM, Ireton RC, Reynolds AB. Monoclonal antibodies to Kaiso: a novel transcription factor and p120 ${ }^{\mathrm{ctn}}$-binding protein. Hybridoma. 2001;20(3):159-66

29. Dreos $\mathrm{R}$, et al. The eukaryotic promoter Database: expansion of EPDnew and new promoter analysis tools. Nucleic Acids Res. 2015;43(Database issue):D92-6.

30. Li LC, Dahiya R. MethPrimer: designing primers for methylation PCRs. Bioinformatics. 2002;18(11):1427-31.

31. Rice P, Longden I, Bleasby A. EMBOSS: the European molecular biology open software suite. Trends Genet. 2000;16(6):276-7.

32. Saxena MT, et al. Murine notch homologs (N1-4) undergo presenilindependent proteolysis. J Biol Chem. 2001;276(43):40268-73.

33. Dang $L$, et al. Notch3 signaling promotes radial glial/progenitor character in the mammalian telencephalon. Dev Neurosci. 2006;28(1-2):58-69.

34. Gersemann M, Stange EF, Wehkamp J. From intestinal stem cells to inflammatory bowel diseases. World J Gastroenterol. 2011;17(27):3198-203.

35. Dorofeyev AE, et al. Mucosal barrier in ulcerative colitis and Crohn's disease. Gastroenterol Res Pract. 2013;2013:431231.

36. Fre $S$, et al. Notch lineages and activity in intestinal stem cells determined by a new set of knock-in mice. PLoS One. 2011;6(10):e25785.

37. Kazanjian $\mathrm{A}$, et al. Atonal Homolog 1 is required for growth and differentiation effects of notch/ $Y$-secretase inhibitors on normal and cancerous intestinal epithelial cells. Gastroenterology. 2010;139(3):918-28. 928.e1-6.

38. Schröder N, Gossler A. Expression of Notch pathway components in fetal and adult mouse small intestine. Gene Expr Patterns. 2002;2(3-4):247-50.

39. Sander GR, Powell BC. Expression of notch receptors and ligands in the Adult Gut. J Basic Appl Histochem Cytochem. 2004;52(4):509-16.

40. Mumm JS, Kopan R. Notch signaling: from the outside in. Dev Biol. 2000; 228(2):151-65.

41. Daniel JM, et al. The p120 ${ }^{\mathrm{ctn}}$-binding partner Kaiso is a bi-modal DNAbinding protein that recognizes both a sequence-specific consensus and methylated CpG dinucleotides. Nucleic Acids Res. 2002;30(13):2911-9.

42. Prokhortchouk A, et al. The p120 catenin partner Kaiso is a DNA methylation-dependent transcriptional repressor. Genes Dev. 2001;15(13): $1613-8$

43. Filion GJ, et al. A family of human zinc finger proteins that bind methylated DNA and repress transcription. Mol Cell Biol. 2006:26(1):169-81.

44. Jiang $\mathrm{G}$, et al. P120-catenin isoforms 1 and 3 regulate proliferation and cell cycle of lung cancer cells via $\beta$-catenin and Kaiso respectively. PLoS One. 2012;7(1):e30303. 
45. Arcaroli JJ, et al. A NOTCH1 gene copy number gain is a prognostic indicator of worse survival and a predictive biomarker to a Notch1 targeting antibody in colorectal cancer. Int J Cancer. 2016;138(1):195-205.

46. Gao J, et al. Up-regulated expression of Notch1 and Jagged1 in human colon adenocarcinoma. Pathol Biol. 2011;59(6):298-302.

47. Reedijk M, et al. Activation of Notch signaling in human colon adenocarcinoma. Int J Oncol. 2008;33(6):1223-9.

48. Rodova M, et al. Regulation of the Rapsyn promoter by Kaiso and $\delta$-catenin. Mol Cell Biol. 2004;24(16):7188-96.

49. Bassey-Archibong Bl, et al. Kaiso depletion attenuates transforming growth factor- $\beta$ signaling and metastatic activity of triple-negative breast cancer cells. Oncogene. 2016;5:e208

50. Koh DI, et al. KAISO, a critical regulator of p53-mediated transcription of CDKN1A and apoptotic genes. Proc Natl Acad Sci U S A. 2014;111(42): 15078-83.

51. Rosenbloom KR, et al. ENCODE Data in the UCSC Genome Browser: year 5 update. Nucleic Acids Res. 2013:41(Database issue):D56-63.

52. Obata Y, et al. Epithelial cell-intrinsic Notch signaling plays an essential role in the maintenance of gut immune homeostasis. J Immunol. 2012;188(5): 2427-36.

53. VanDussen $\mathrm{KL}$, et al. Notch signaling modulates proliferation and differentiation of intestinal crypt base columnar stem cells. Development. 2012;139(3):488-97.

54. van Es JH, et al. Notch/Y-secretase inhibition turns proliferative cells in intestinal crypts and adenomas into goblet cells. Nature. 2005;435(7044): 959-63.

55. Prokhortchouk AV, et al. KAISO-a new member of the BTB/POZ family specifically bindsto methylated DNA sequences. Genetika. 2001;37(6):737-44.

56. Spring $\mathrm{CM}$, et al. The catenin p120 ${ }^{\mathrm{ctn}}$ inhibits Kaiso-mediated transcriptional repression of the $\beta$-catenin/TCF target gene matrilysin. Exp Cell Res. 2005; 305(2):253-65

57. Sasai N, Nakao M, Defossez PA. Sequence-specific recognition of methylated DNA by human zinc-finger proteins. Nucleic Acids Res. 2010;38(15):5015-22.

58. Vermeulen JF, et al. Nuclear Kaiso expression is associated with high grade and triple-negative invasive breast cancer. PLoS One. 2012;7(5):e37864

59. Jones J, et al. Nuclear Kaiso indicates aggressive prostate cancers and promotes migration and invasiveness of prostate cancer cells. Am J Pathol. 2012;181(5):1836-46

\section{Submit your next manuscript to BioMed Central and we will help you at every step:}

- We accept pre-submission inquiries

- Our selector tool helps you to find the most relevant journal

- We provide round the clock customer support

- Convenient online submission

- Thorough peer review

- Inclusion in PubMed and all major indexing services

- Maximum visibility for your research

Submit your manuscript at www.biomedcentral.com/submit

C) Biomed Central 